Design for a High Energy Density Kelvin-Helmholtz Experiment

O. A. Hurricane

November 1, 2007

High Energy Density Physics 
This document was prepared as an account of work sponsored by an agency of the United States government. Neither the United States government nor Lawrence Livermore National Security, LLC, nor any of their employees makes any warranty, expressed or implied, or assumes any legal liability or responsibility for the accuracy, completeness, or usefulness of any information, apparatus, product, or process disclosed, or represents that its use would not infringe privately owned rights. Reference herein to any specific commercial product, process, or service by trade name, trademark, manufacturer, or otherwise does not necessarily constitute or imply its endorsement, recommendation, or favoring by the United States government or Lawrence Livermore National Security, LLC. The views and opinions of authors expressed herein do not necessarily state or reflect those of the United States government or Lawrence Livermore National Security, LLC, and shall not be used for advertising or product endorsement purposes. 


\title{
Design for a High Energy Density Kelvin-Helmholtz Experiment
}

\author{
O. A. Hurricane \\ Lawrence Livermore National Laboratory \\ P.O. Box 808, Livermore, CA 94551 \\ (UCRL-JRNL-XXXXXX, February 2007)*
}

\begin{abstract}
While many high energy density physics (HEDP) Rayleigh-Taylor and Richtmyer-Meshkov instability experiments have been fielded as part of basic HEDP and astrophysics studies, not one HEDP Kelvin-Helmholtz (KH) experiment has been successfully performed. Herein, a design for a novel HEDP x-ray driven KH experiment is presented along with supporting radiation-hydrodynamic simulation and theory.

PACS numbers: 52.58.Lq,52.59.Hq,52.65.Kj
\end{abstract}

\section{INTRODUCTION}

Motivated by the need to better understand the instability processes that occur in inertial confinement fusion (ICF) and supernovae, and the need to gauge the quality of the computer simulations that are used to model the these processes, the field of high energy density physics (HEDP) has developed. HEDP experiments, used to compare to simulation predictions, characteristically deposit 10's of kiloJoules of energy in millimeter scale targets on timescales of nanoseconds thus generating plasmas at megabar pressures. The resulting flows emulate, by design, important unstable processes and can often be self-similarly scaled to astrophysics contexts [1-4]. A number of experiments that explore acceleration driven Rayleigh-Taylor instability [5] and shock driven Richtmyer-Meshkov instability [6] have been explored previously. While a shear flow driven experiment has been attempted [7], whether Kelvin-Helmholtz instability actually resulted was not evident.

In 2002, a very elegant Kelvin-Helmholtz target was designed for the National Ignition Facility (NIF) Early Light (NEL) experiment. In fact, this design was the leading candidate for NEL until it became clear that the NEL configuration could not accommodate the long backlighter delay required for the experiments success. The key feature to the design, was to utilize the large vorticity generation mechanism that is associated with maximizing the cross product of pressure gradient and a density gradient (baroclinic vorticity production) that occurs when a shock wave passes laterally across a perturbed material interface, that is, with shock velocity perpendicular to the surface normal. While this design generated a great deal of interest at the time, as calculations showed that it was not only scientifically valuable but visually impressive, the design has been filed away since 2003, to be fielded at a future date.

The basic configuration consists of a sandwich of an opaque high density plastic $\left(\mathrm{CHBr}_{4.3 \%}\right)$ and a low density CRF foam all of which is contained in a rectangular cross-section shock tube, made from Be in order to radio- graph through it (see Fig. 1). In the original NEL design, the laser energy (a "quad" of NIF at energy $7.5 \mathrm{~kJ}$ in a 1.25 ns pulse @ $3 \omega$ ) would be delivered to a 1 square mm spot on an ablator covering the low density foam part of the target. In this way, a strong shock is launched into the low density foam such that the pressure gradient at the leading edge of the shock would essentially be a right angles to the density gradient at the interface of the two dissimilar materials. The interface between the two materials is perturbed by a sinusoidal contour with amplitude $(a)$ and wavelength $(\lambda)$ chosen such that a number of large vortices's would develop nonlinear structure in the expected field of view in the duration of the experiment (limited by the backlighter delay time). In the case of Fig. $1, a=60 \mu \mathrm{m}$ and $\lambda=400 \mu \mathrm{m}$. The asymmetric target design was selected to maximize the amount of laser energy that could be delivered to the low density foam. For Fig. 1 the target length is $4 \mathrm{~mm}$ and the total target height is $2.0 \mathrm{~mm}$.

\section{DESIGN THEORY}

From the curl of the momentum equation, the vorticity equation is,

$$
\frac{d \boldsymbol{\omega}}{d t}+\boldsymbol{\omega} \boldsymbol{\nabla} \cdot \mathbf{v}+\boldsymbol{\omega} \cdot \nabla \mathbf{v}=\frac{\boldsymbol{\nabla} \rho \times \nabla P}{\rho^{2}}
$$

where $d / d t$ is the advective time derivative and $\boldsymbol{\omega}=\boldsymbol{\nabla} \times$ $\mathbf{v}$ is the vorticity. In situations where the velocity is invariant along a coordinate direction, the third term on the left vanishes identically. Thus the two dimensional (2D) vorticity equation is,

$$
\frac{d \boldsymbol{\omega}}{d t}+\omega \nabla \cdot \mathbf{v}=\frac{\nabla \rho \times \nabla P}{\rho^{2}}
$$

the baroclinic vorticity term being the term on the right hand side.

Since we are interested in dynamics on timescales that are long compared to the time it takes the shockwave to transit over a wavelength of the perturbation between 


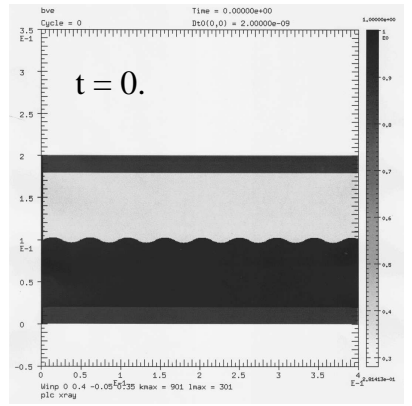

(a)

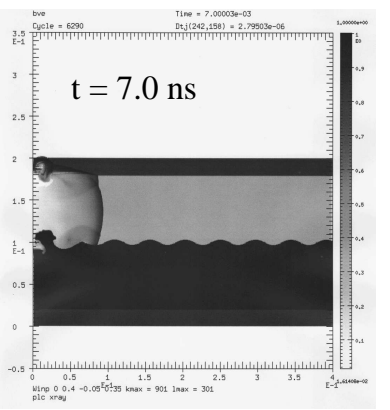

(b)
FIG. 1: (a) The $t=0$ geometry of the target as seen in a synthetic x-ray image. The high density $\mathrm{CHBr}_{4.3 \%}$ is the dark region on the bottom half of the frame, while the low density foam is on the top half. The wall of the beryllium shock tube bounding the foam is clearly visible on top, while the same wall on the bottom is harder to see, but present. A $30 \mu \mathrm{m}$ plastic ablator is barely visible as a dark line on the left of the target. (b) Early in the targets evolution, at $t=7$ $\mathrm{ns}$, the shock generated by the laser pulse is moving from left to right at $7.5 \mathrm{~cm} / \mu \mathrm{s}$. The pressure gradient across the shock, which at this time is 6.5 Mbars, is nearly orthogonal to the density gradient as designed.

the material interfaces, it is appropriate to treat the baroclinic vorticity generation term as a source term at $t=0$. That is, any subsequent gradients in density or pressure that might produce vorticity will be treated as insignificant compared to the vorticity generated by the passage of the shockwave.

In order to model the baroclinic vorticity term, the shock wave is treated as a discrete jump pressure moving at the shock speed, and the perturbed material interface is treated as a discrete jump in density (see Fig. 2). That is,

$$
\begin{array}{r}
P=\Delta P \mathcal{H}\left(u_{s} t-x\right)+P_{0} \\
\rho=\left(\rho_{L}-\rho_{H}\right) \mathcal{H}\left(y-y_{I}(x)\right)+\rho_{H}
\end{array}
$$

where $P_{0}$ is the pressure in the low density $\left(\rho_{L}\right)$ material before the passage of the shock, $\Delta P$ is the pressure jump of the shock front, $u_{s}$ is the shock speed, $y_{I}(x)$ is the contour of the perturbed interface between the low and high density $\left(\rho_{H}\right)$ materials, and $\mathcal{H}$ is the Heaviside step function.

Assuming that the imposed perturbation of the interface is $y_{I}=y_{0}+a \sin (k x)$, where the wavelength of the perturbation is $\lambda=2 \pi / k$, it is straight forward to show that the unit normal to the interface is given by

$$
\hat{\boldsymbol{n}}=\frac{\hat{\boldsymbol{y}}-k a \cos (k x) \hat{\boldsymbol{x}}}{\sqrt{1+(k a)^{2} \cos ^{2}(k x)}} .
$$

The initial gradients of pressure and density can now be expressed as,

$$
\begin{array}{r}
\boldsymbol{\nabla} \rho=\left(\rho_{L}-\rho_{H}\right) \delta\left(y-y_{I}(x)\right) \hat{\boldsymbol{n}} \\
\nabla p=\Delta P \delta\left(u_{s} t-x\right) \hat{\boldsymbol{x}}
\end{array}
$$

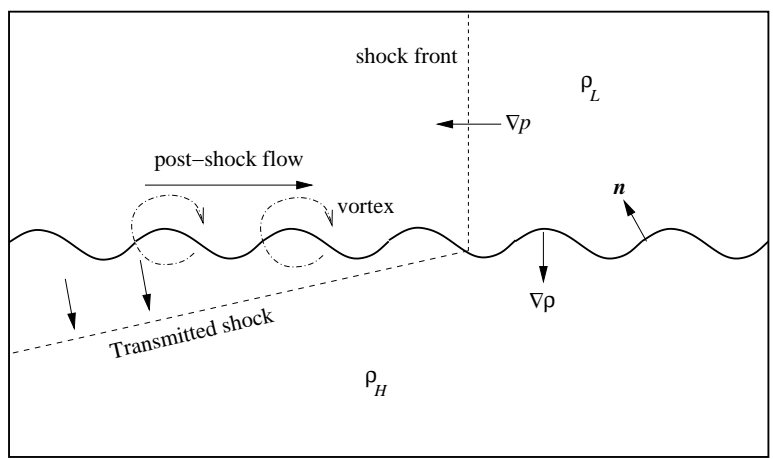

FIG. 2: A laser driven shock travels to the right in the low density material creating a moving pressure jump. This shock traverses a perturbed interface at nearly right angles to the density gradient. It is unavoidable that a shock is transmitted into the high density material, but at an angle such that the transmitted pressure gradient forms a small angle with the interfaces density gradient.

where $\delta$ is the usual Dirac $\delta$-function. Eqs. [6] and [7] are needed to construct the baroclinic vorticity term in Eq. [2].

In order to calculate the rate at which we expect the interface vortex to overturn, the circulation, $\Gamma=\oint \boldsymbol{\omega} \cdot d \mathbf{A}$ (the integral being over area) is a convenient concept. Using Eqs. [2], [6], and [7] along with the definition of the circulation we then have

$$
\Gamma=\int \frac{\Delta \rho \delta\left[y-y_{I}(x)\right] \Delta P \delta\left(u_{s} t-x\right) d x d y d t}{\left[\Delta \rho H\left(y-y_{I}(x)\right)+\rho_{H}\right]^{2} \sqrt{1+(k a)^{2} \cos ^{2}(k x)}} .
$$

where $\Delta \rho=\rho_{H}-\rho_{L}$. Upon performing the spatial integrations one finds

$$
\Gamma=\frac{4 \mathcal{A} \Delta P}{\left(\rho_{H}+\rho_{L}\right)} \int_{0}^{2 \pi /\left(k u_{s}\right)} \frac{d t}{\sqrt{1+(k a)^{2} \cos ^{2}\left(k u_{s} t\right)}},
$$

where $\mathcal{A}=\Delta \rho /\left(\rho_{H}+\rho_{L}\right)$ is the Atwood number. The time integral solution of Eq. [9] is a special function, the Elliptic-K function,

$$
\Gamma=\frac{16 \mathcal{A} \Delta P}{\left(\rho_{H}+\rho_{L}\right)} \frac{K\left(\frac{k a}{\sqrt{1+(k a)^{2}}}\right)}{k u_{s} \sqrt{1+(k a)^{2}}} .
$$

Eq. (10) is the circulation per vortex. Equating Eq.(10) to the alternate definition of vorticity $\oint \mathbf{v} \cdot d \mathbf{l}$ allows one to calculate the eddy turnover speed and therefore eddy turnover frequency, $\Omega_{B V E}=k^{2} \Gamma / 2 \pi^{3}$, by assuming that the diameter of the vortex is the wavelength of the initial perturbation. For the parameters of the simulation (see the next section), $\Delta P \sim 1.3 \mathrm{Mb}, \rho_{L} \sim 0.1$ $\mathrm{g} / \mathrm{cc}, \rho_{H} \sim 1.4 \mathrm{~g} / \mathrm{cc}$, and $v \sim 3.5 \mathrm{~cm} / \mu$ s one finds $\Omega_{B V E} \sim 2.8$ revolutions in $100 \mathrm{~ns}$ consistent with the observed behavior of the simulation.

As can be found in many classical fluid dynamics books [9] the complex potential can be related to the vorticity 
through the residue theorem. For an array of infinite vortex lines the complex potential is [10]

$w(z)=\sum_{n=-\infty}^{\infty}\left(i \frac{\Gamma}{2 \pi}\right) \ln \left(z-n \lambda+\frac{\lambda}{2}\right)=\frac{i \Gamma}{2 \pi} \ln \sin \left(\frac{\pi z}{\lambda}+\frac{\pi}{2}\right)$

where $z=x+i y$ is the complex coordinate and $v_{x}-i v_{y}=$ $d w / d z$ relates the components of the flow velocity to the complex potential. The differential equations for the flow field are then

$$
\begin{aligned}
& \frac{d x}{d t}=\frac{\Gamma}{4 \lambda} \frac{\sinh \left(\frac{2 \pi y}{\lambda}\right)}{\cos ^{2}\left(\frac{\pi x}{\lambda}\right)+\sinh ^{2}\left(\frac{\pi y}{\lambda}\right)} \\
& \frac{d y}{d t}=\frac{\Gamma}{4 \lambda} \frac{\sin \left(\frac{2 \pi x}{\lambda}\right)}{\cos ^{2}\left(\frac{\pi x}{\lambda}\right)+\sinh ^{2}\left(\frac{\pi y}{\lambda}\right)} .
\end{aligned}
$$

Taylor expanding Eqs. (12) and (13) about $x=0$ and $y=0$, retaining the leading term of each, and eliminating $x$ in the expressions results in a simple differential equation $d^{2} y / d t^{2}=\gamma_{B V E}^{2} y$, where $\gamma_{B V E}=k^{2} \Gamma / 8 \pi$ is the linear growth rate. The typical linear growth rate for Kelvin-Helmholtz instability is $\gamma_{K H}=\sqrt{\rho_{H} \rho_{L}} k v /\left(\rho_{H}+\right.$ $\left.\rho_{L}\right)$ which is clearly different than $\gamma_{B V E}$ hence the distinction. That is, Taylor expanding Eq.[10] in $k a$ and applying the Rankin-Hugoniot shock relations one finds

$\frac{\gamma_{B V E}}{\gamma_{K H}} \approx \frac{2}{\gamma_{L}+1} \mathcal{A} \sqrt{\frac{\rho_{L}}{\rho_{H}}} \frac{c_{H}}{c_{L}}\left[1-\frac{1}{4}(k a)^{2}+\frac{9}{64}(k a)^{4}+\ldots\right]$

where $\gamma_{L(H)}$ is the polytropic index of the low (high) density material and $c_{L(H)}$ is the pre-shock sound speed of the low (high) density material.

Taking the ratio of Eqs. (13) and (12) yields an equation for $d y / d x$ that is separable and trivially solvable one obtains

$\cosh \left(\frac{2 \pi y}{\lambda}\right)-\cosh \left(\frac{2 \pi y_{0}}{\lambda}\right)=\cos \left(\frac{2 \pi x_{0}}{\lambda}\right)-\cos \left(\frac{2 \pi x}{\lambda}\right)$.

Eq. (15) describes, for all time, the "orbit" that a massless particle suspended in the fluid would take when starting out at position $\left(x_{0}, y_{0}\right)$ at $t=0$. A plot of the "phase portrait" of the differential equations Eqs. (12) and (13) is provided by Eq. (15) and is shown in Fig. 3. A separatrix bounds an inner flow region where fluid continually overturns and an outer transiting flow. The equation describing the separatrix is given by Eq. (15) with $x_{0}=0$ and $y_{0}=0$ from which one can find that the maximum $y$ extent of the overturning region is $\cosh ^{-1}(3) \lambda /(2 \pi) \approx 0.281 \lambda$.

\section{DESIGN SIMULATION}

Simulations of the proposed target were performed with the Lawrence Livermore National Laboratory

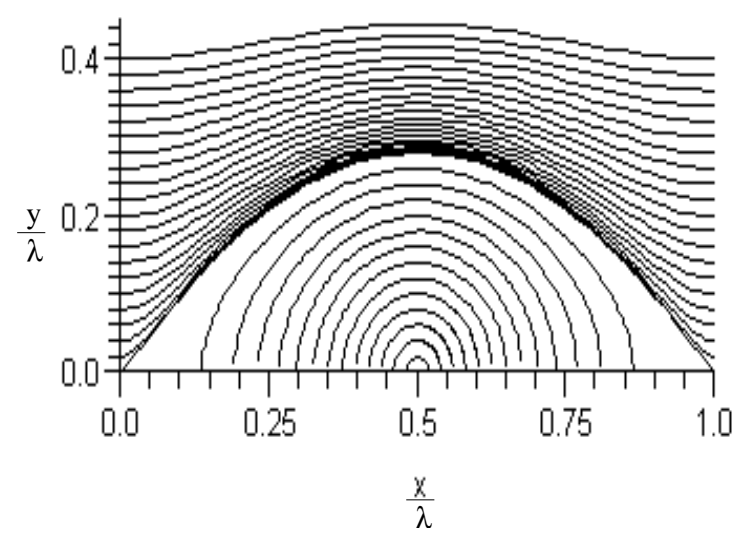

FIG. 3: The figure displays the phase portrait given by Eq. (15). The outer flowing region is clearly distinct from the inner vortex region. The form of this phase portrait is independent of time. The rate that the vortex overturns is directly proportional to the circulation, $\Gamma$, which interestingly is the only place where the amplitude of the perturbation comes into play.

(LLNL) code CALE (C-based Arbitrary Lagrangian Eulerian) [8]. The simulation includes direct laser deposition of energy at a surface determined by the electron plasma frequency, tabulated material equations of state, tabulated opacity, ionization, electron heat conduction, and radiation diffusion. The laser source intensity is prescribed by a super-Gaussian profile $I(y)=$ $I_{0} \exp \left[-\left(y-y_{0}\right) / \sigma\right]^{N}$, with $N=5$ and $\sigma=400 \mu \mathrm{m}$ (ranges of $N=3-7$ and $\sigma=300-500 \mu \mathrm{m}$ were simulated to test target sensitivity and none was observed).

In CALE, hydrodynamics are solved at second order accuracy except at shocks where the accuracy is first order. The mesh in the initial setup of the problem is conformal to the material interfaces, but as the problem evolves materials will eventually advect through the mesh. That is, the simulation starts off Lagrangian with the mesh following the flows, but inevitably as the mesh distorts, material is allowed to partially slip through the mesh in a more Eulerian sense. In the simulations shown here, the resolution is 901 zones along $x$ and 301 zones along $y$. At lower resolutions the desired vortex structure is numerically diffused away in CALE.

The six frames of Fig. 4 show the evolution of the target as would be seen radiographically (synthetic radiograph images are calculated using the cold material opacities at the photon frequency of an iron backlighter). The first frame shows the state of the target at $t=10$ ns, where the shock has only crossed over the first two wavelengths of the perturbation. At $t=10 \mathrm{~ns}$, the second wavelength of the perturbation has sharpened into a wave that is on the verge of breaking. The next five frames of the figure show the target state at 20,30,40, 50 , and $60 \mathrm{~ns}$. As the shock moves downs the target shock 

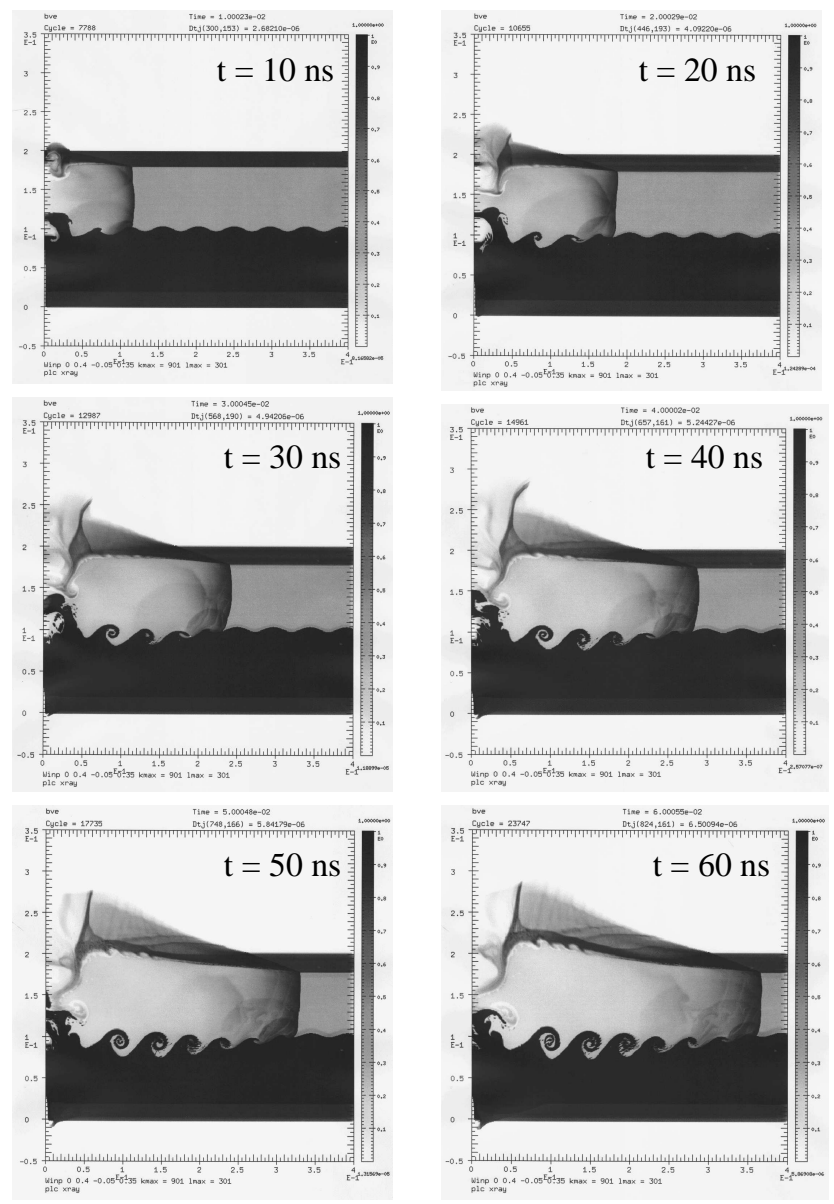

FIG. 4: The six frames show the target evolution, in synthetic radiograph, from $t=10 \mathrm{~ns}$ to $t=60 \mathrm{~ns}$ in $10 \mathrm{~ns}$ increments. As the main shock in the low density foam moves down the target from left to right, cylindrical shock reflections are produced at the perturbed interface. These shock reflections propagate upward through the post-shock foam and imprint structure on the Be shock tubes inner surface. After the passage of the main shock, it takes about $20 \mathrm{~ns}$ for the vortex structure of a wavelength of the perturbation to grow into a distinct roll-up.

reflections are observed in the post-shock flow in the low density foam. These reflections imprint structure on the inner wall of the beryllium shock tube. Vortex structures are evident by $30 \mathrm{~ns}$. The center of each vortex is centered on the locations of the crests of the imposed perturbation with the diameter of each vortex corresponding to the wavelength of the initial perturbation.

By the time the main flow-driving shock exits the target on the right (Fig. 5), at $t=74$ ns the first distinct vortex has turned over approximately 3 times. Due to the flow pattern setup by the shock that was transmitted into the high density plastic, the vortex is stretched in $y$ as it evolves which is why the extent of the vortex, in $y$, is larger than the $0.281 \lambda$ analytic result following Eq. (15).

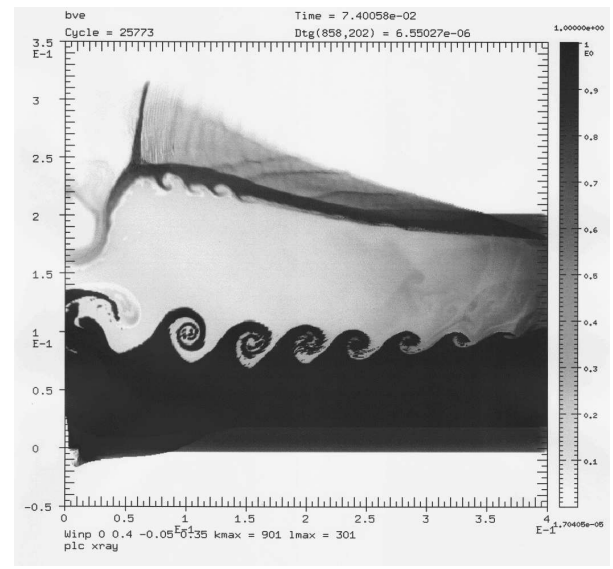

FIG. 5: By the time the main shock exits the target on the right (74 ns), the left-most vortex structure has overturned about 3 times, while the right-most is just starting to spin. In this way, a "snap-shot" of the entire target at this stage reveals the entire temporal vortex evolution.

Based upon calculation of the Reynolds number, the vortex sheet shown in Fig. 5, may transition into turbulence in a few eddy turn-over times. Using the Spitzer ion-ion collision frequency, $\nu_{i i}=$ $\sqrt{2} \pi Z^{4} e^{4} n_{i} \ln \Lambda / \sqrt{m_{i} T_{i}^{3}}$ one may estimate the viscosity $\nu=v_{t}^{2} /\left(3 \nu_{i i}\right)$, where $Z$ is the average atomic charge, $e$ is the electron charge, $n_{i}$ is the average ion density, $m_{i}$ is the average ion mass, $T_{i}$ is the ion temperature, and $v_{t}$ is the ion thermal speed. One obtains $\nu \sim 3 \times 10^{-6} \mathrm{~cm}^{2} / \mathrm{s}$. Using a length-scale, $L \sim 10 \mu \mathrm{m}$, indicative of the thickness of the arms of the wound vortex, one finds that the Reynolds number, $R_{e}=L v / \nu \sim 3 \times 10^{6}$ which is a value that is characteristic of the transition to turbulence.

\section{CONCLUSION}

Arguably, shear-flow instabilities are a stiffer test of computer simulations than buoyancy driven instabilities especially for simulations that have a Lagrangian nature in that mesh follows the flow. HEPD experiments on Kelvin-Helmholtz can also provide much needed data for the development of turbulent mix models that attempt to address shear-flow driven turbulence production.

The experimental design studied in this paper is intriguing since it appears to generate a array of vortices's that are large and therefore diagnosable. With such experiments, it may be possible to directly observe transitions to turbulence or deeply non-linear vortex merger effects [11].

Another interesting possible extension of this platform involves magnetic field (B) production - namely the Biermann Battery effect. In the limit where electron inertial can be neglected, electrons obey the equation of motion $\boldsymbol{\nabla} p_{e}=-|e| n_{e}\left(\mathbf{E}+\mathbf{v}_{\mathbf{e}} \times \mathbf{B}\right)$, where $p_{e}$ is electron pressure, 
$e$ is the electron charge, $n_{e}$ is the electron density, $\mathbf{E}$ is the electric field, and $\mathbf{v}_{\mathbf{e}}$ is the electron velocity. When combined with Faraday's law of induction one finds and equation for magnetic field generation,

$$
\frac{\partial \mathbf{B}}{\partial t}+\mathbf{v}_{\mathbf{e}} \cdot \boldsymbol{\nabla} \mathbf{B}+\mathbf{B} \boldsymbol{\nabla} \cdot \mathbf{v}_{\mathbf{e}}=\mathbf{B} \cdot \boldsymbol{\nabla} \mathbf{v}_{\mathbf{e}}+\frac{\boldsymbol{\nabla} T_{e} \times \boldsymbol{\nabla} n_{e}}{|e| n_{e}}
$$

where $T_{e}$ is the electron temperature. What should be noted is that Eq. [16] is almost identical in form to Eq. [1] suggesting the possibility that this proposed experiment may also produce significant magnetic field.

Thanks to Dr. Harry Robey for his input into the design of this experiment. The encouragement of Dr.'s Bruce Remington and J. Freddy Hansen is greatly appreciated. Also thanks to Prof. R. Paul Drake and his student Eric C. Harding for their interest in ressurecting this experimental proposal. This work was performed under the auspices of the U.S. Department of Energy by the University of California Lawrence Livermore National Laboratory under contract No. W-7405-Eng-48.

Prepared by LLNL under Contract DE-AC5207NA27344

* Electronic address: hurricane1@llnl.gov

[1] B.A. Remington, R.P. Drake, D. Arnett, and H. Takabe, Science, 284, 1488, 1999.

[2] D.D. Ryutov, R.P. Drake, J. Kane, E. Liang, B.A. Remington, and M. Wood-Vasey, Astrophys. J., 518, 821,
1999.

[3] R.P. Drake, J.J. Carroll III, T.B. Smith, P. Keiter, S.G. Glendinning, O. Hurricane, K. Estabrook, D.D. Ryutov, B.A. Remington, R.J. Wallace, E. Michael, R. McCray, Phys. Plasmas, 7, 2142, 2000.

[4] H.F. Robey, J.O. Kane, B.A. Remington, R.P. Drake, O.A. Hurricane, H. Louis, R.J. Wallace, J. Knauer, P. Keiter, D. Arnett, and D.D. Ryutov, Phys. Plasmas, 8, 2446, 2001.

[5] R.P. Drake, H.F. Robey, O.A. Hurricane, Y. Zhang, B.A. Remington, J. Knauer, J. Glimm, D. Arnett, J.O. Kane, K.S. Budil, and J. Grove, Astrophys. J., 564, 2002.

[6] J.O. Kane, H.F. Robey, B.A. Remington, R.P. Drake, J. Knauer, D.D. Ryutov, H. Louis, R. Teyssier, O. Hurricane, D. Arnett, R. Rosner, and A. Calder, Phys. Rev. E, 63, 055401, 2001.

[7] B.A. Hammel, J.D. Kilkenny, D. Munro, B.A. Remington, H.N. Kornblum, T.S. Perry, D.W. Phillion, and R.J. Wallace, Phys. Plasmas, 1, 1662, 1994.

[8] R. T. Barton, "Development of a multimaterial twodimensional, arbitrary Lagrangian-Eulerian mesh computer program," in Numerical Astrophysics, J.M. Centrella, J.M. LeBlanc, R.L. Bowers, eds., (Jones and Bartlett Publishers, Boston, 1985), p. 482.

[9] L. D. Landau and E. M. Lifshitz, "Fluid Mechanics (2nd Ed.)," (Pergamon Press, Oxford, 1989), p. 20.

[10] A. R. Paterson, "A First Course in Fluid Dynamics," (Cambridge Univ. Press, 1983), p. ?.

[11] A. Rikanati, D. Oron, U. Alon, and D. Shvarts, Astrophys. J., 127, 451, 2000. 